\title{
Cardiac involvement in ERdheim-Chester disease: a CASE REPORT
}

\author{
Fernando Pivatto Júnior ${ }^{1}$, Diogo Silva Piardi ${ }^{1}$, Felipe Soares Torres ${ }^{2}$
}

Clin Biomed Res. 2014:34(4):406-409

1 Cardiology Division, Hospital de Clínicas de Porto Alegre. Porto Alegre, RS, Brazil.

2 Radiology Department, Hospital de Clínicas de Porto Alegre. Porto Alegre, RS, Brazil.

Corresponding author: Fernando Pivatto Júnior

E-mail: fpivatto@gmail.com Cardiology Division

Hospital de Clínicas de Porto Alegre Rua Ramiro Barcelos, 2350. 90035-903, Porto Alegre, RS, Brazil.

\section{ABSTRACT}

Erdheim-Chester disease (ECD) is a rare non-Langerhans cell histiocytosis of unknown etiology characterized by proliferation of lipid-containing foamy histiocytes affecting bones and potentially every organ. There is a wide clinical spectrum of the disease, ranging from asymptomatic bone or soft-tissue lesions to life-threatening systemic involvement. Although the initial descriptions published by Jakob Erdheim and William Chester in 1930 included a patient with right atrial infiltration on autopsy, cardiovascular involvement in ECD has only gained more recognition recently. In the present report, we describe a case with cardiac involvement, presenting with symptomatic heart failure and a cardiac mass assessed with echocardiogram and cardiac magnetic resonance imaging.

Keywords: Erdheim-Chester disease; heart failure; magnetic resonance imaging

\section{CASE REPORT}

A 50-year-old female patient, previously asymptomatic, with hypertension, type-2 diabetes, chronic kidney disease, and Erdheim-Chester disease (ECD) was admitted due to progressive dyspnea (NYHA class III), orthopnea, and paroxysmal nocturnal dyspnea, which started one month earlier. ECD diagnosis was established seven years earlier after the results of a biopsy of a bone lesion in the right tibia. In addition, the patient had been diagnosed with retroperitoneal fibrosis and eye xanthogranuloma, both related to ECD, and had been treated with prednisone and interferon- $\alpha$ since the diagnosis.

Physical examination upon admission showed basilar pulmonary rales and ankle edema. The electrocardiogram (ECG) is shown in Figure 1. Transthoracic echocardiography was performed (figure 2) and showed an ejection fraction of $47 \%$, eccentric hypertrophy, mild diffuse hypokinesia, thickening of the interatrial septum and of the posterior wall of the right atrium, referred as lipomatous hypertrophy of the interatrial septum, and a large pericardial effusion, without signs of high intrapericardial pressure. The patient showed good symptomatic response to beta-blocker, furosemide, isosorbide dinitrate/ hydralazine, and amlodipine and was discharged.

Cardiac magnetic resonance imaging (MRI) was performed (figure 3) to assess the left atrium and interatrial septum and showed abnormal soft tissue thickening of the posterior wall of the right atrium and of the right atrioventricular groove, consistent with cardiac involvement of ECD. In addition, there was significant reduction in the volume of the pericardial effusion, most likely secondary to the pharmacological treatment of heart failure. Because of the typical appearance of the cardiac MRI findings, a cardiac biopsy was deemed unnecessary.

\section{DISCUSSION}

ECD is a rare non-Langerhans cell histiocytosis of unknown etiology characterized by proliferation of lipid-containing foamy histiocytes affecting bones and potentially every organ ${ }^{1}$. The diagnosis is based on the presence of symmetric bilateral osteosclerosis of the metaphysis and diaphysis of long 


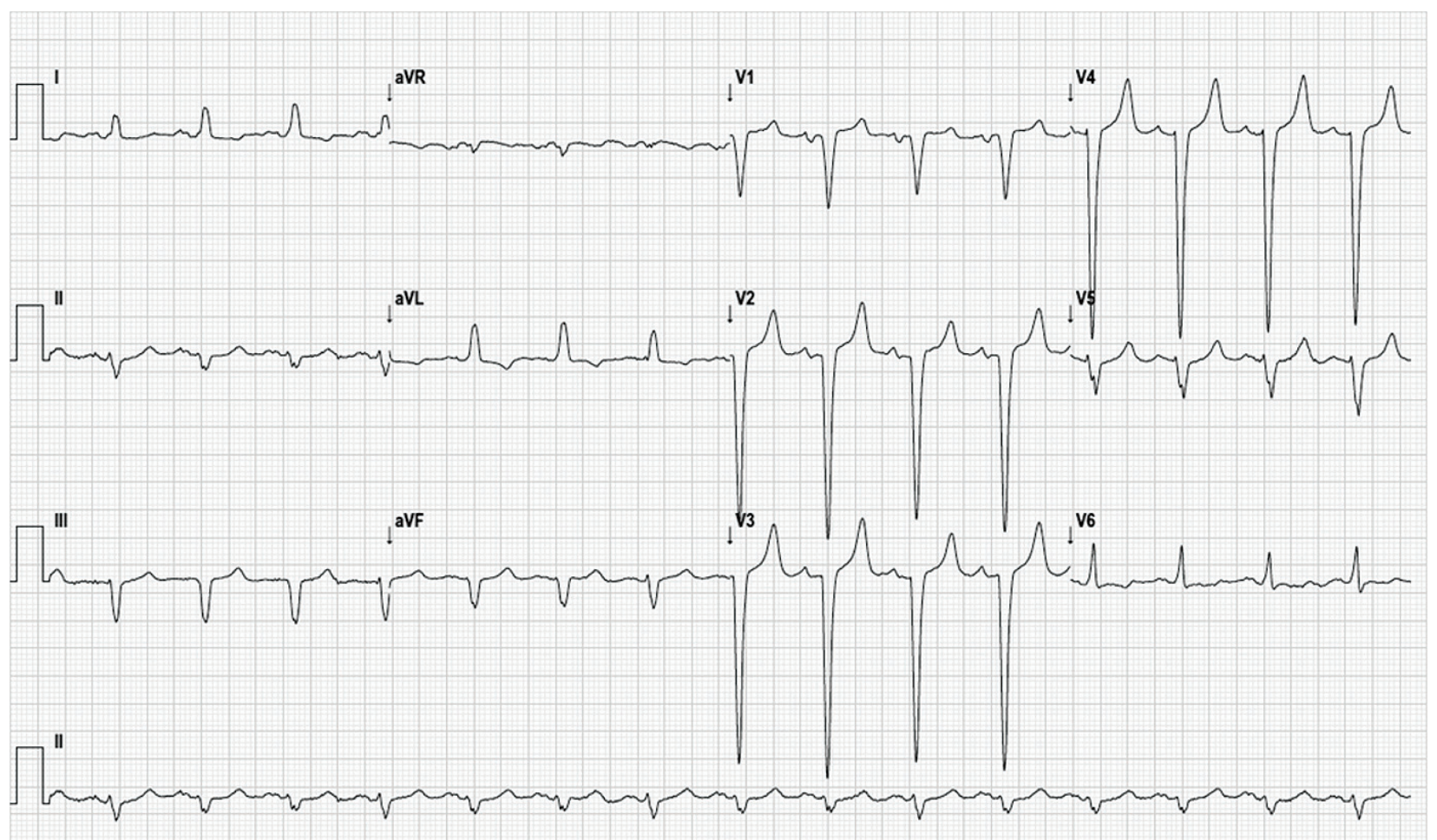

Figure 1: Twelve-lead ECG showed left atrial and ventricular hypertrophy with secondary repolarization changes.

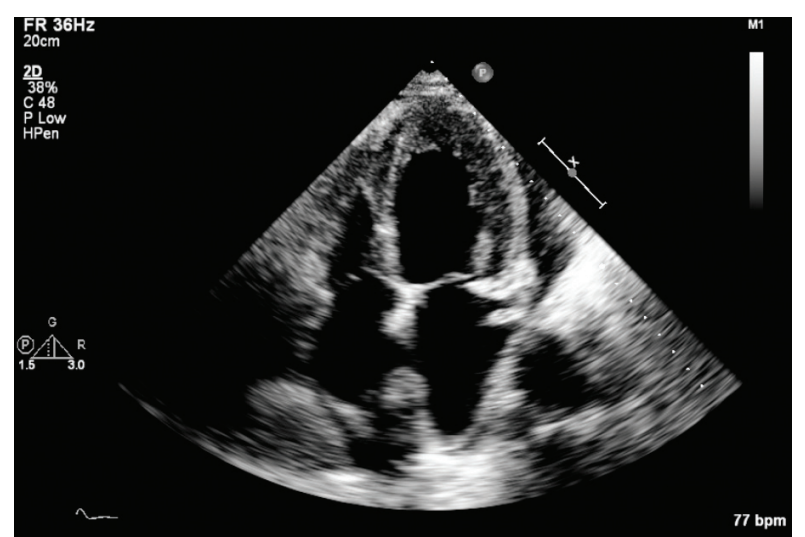

Figure 2: Transthoracic echocardiography apical 4-chamber view showing thickening of the interatrial septum and of the posterior wall of the right atrium, referred as lipomatous hypertrophy of the interatrial septum.

bones, and confirmed by histologic examination showing foamy histiocytes CD68+, PS100+/-, CD1a-2 .

Its broad clinical spectrum ranges from asymptomatic bone or soft-tissue lesions to life-threatening systemic involvement ${ }^{1}$. Clinical manifestations may be protean and are attributable to the functional compromise of organs by histiocytic infiltration leading to arterial constriction, which results in end-organ atrophy or failure. ECD frequently involves the appendicular skeletal bones. Therefore, bone pain is the most frequent symptom because of the formation of osteosclerotic lesions of long bones). This was the case of our patient ${ }^{3}$. Approximately half of patients have extra skeletal manifestations, including exophthalmos, xanthelasma, interstitial lung disease, retroperitoneal fibrosis with perirenal or ureteral obstruction, renal failure, diabetes insipidus, and central nervous system and cardiovascular involvement ${ }^{4}$. Although the first descriptions published by Jakob Erdheim and William Chester in 1930 included a patient with right atrial myocardial infiltration on autopsy, only recently cardiovascular involvement in ECD started to gain more recognition. Originally thought to be uncommon, cardiovascular involvement in ECD occurs in $40 \%$ of cases $^{3}$. Cardiac manifestations of ECD include pericardial infiltration, myocardial infiltration, right atrial involvement, and symptomatic valvular heart disease. The most common finding reported is infiltration of the right heart (49\%), with approximately $30 \%$ of these cases being attributed to tumefactive infiltration of the right atrium. Other less commonly reported findings include atrioventricular sulcus infiltration, pericardial effusion (with pericardial thickening), and periarterial infiltration of the coronary arteries. More than half of patients have periaortic fibrosis ${ }^{5}$. When the entire 

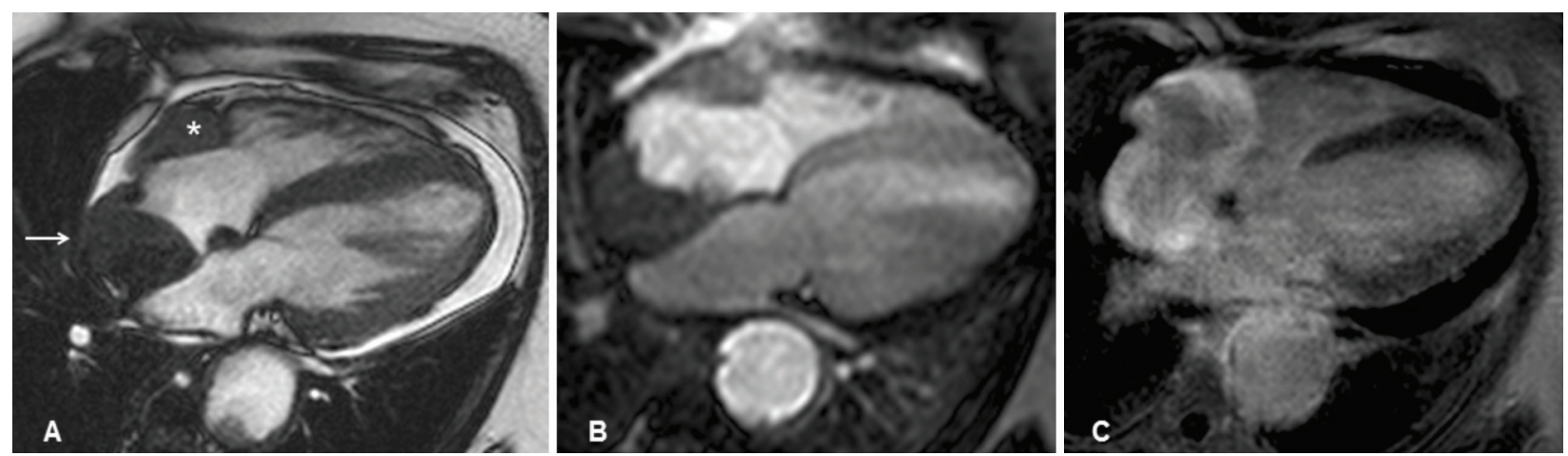

Figure 3: (A) Balanced, steady-state, free-precession, still-frame from 4-chamber view showing abnormal soft tissue thickening of the posterior wall of the right atrium (arrow) and of the right atrioventricular groove $\left(^{*}\right)$. (B) First-pass myocardial perfusion apical 4-chamber view showing less intense myocardial perfusion of the soft tissue thickening in comparison with the ventricular myocardium. (C) Late gadolinium enhancement 4-chamber view showing intense and mostly peripheral gadolinium enhancement of the abnormal soft tissue thickening in the posterior wall of the right atrium and right atrioventricular groove.

aorta is involved, computed tomography (CT) may reveal the appearance of a "coated aorta". Infiltration of other arteries may lead to variable clinical ischemic manifestations, such as myocardial infarction, mesenteric angina, cerebral ischemia, and renovascular hypertension ${ }^{3}$.

ECD has a variable clinical course depending on the extent and location of the lesions. Nevertheless, this disease may be devastating when there is cardiac involvement ${ }^{4}$. The prognosis is extremely variable and is often worse when there is a cardiovascular and/or central nervous system involvement ${ }^{2}$. One third of the deaths associated with ECD are caused by cardiovascular disease, including heart failure, myocardial infarction, cardiac tamponade, and aortic occlusion. Therefore, screening for cardiac involvement with cardiac MRI or CT imaging in all patients diagnosed with $E C D$, even in the absence of cardiac symptoms, has been suggested ${ }^{3}$. Treatment is controversial and it is often based on corticosteroids and/or interferon- $\alpha^{2}$. Interferon- $\alpha$ is currently the firstline therapy, but its use requires an individualized approach $^{4}$.

The diagnosis of ECD should be entertained in all patients with intracardiac and pericardial masses, particularly in the right atrium (RA). RA infiltration occurs frequently and may cause formation of a "pseudotumor". The differential diagnosis of RA masses includes, in order of likelihood, myxoma, thrombus, and metastatic and primary malignant cardiac tumors ${ }^{3}$.

Lipomatous hypertrophy of the interatrial septum (LHIS) may be defined as a benign cardiac mass characterized by 2-cm-thick fatty deposits in the interatrial septum 6 . It encompasses a non-capsulated circumscribed, fatty mass and should be considered as part of the differential diagnosis for any atrial cardiac tumor. In this case report, this was the diagnostic hypothesis for the image observed in the echocardiogram. Differentiation of LHIS from other intracardiac masses is almost always possible using conventional imaging techniques, thus limiting the need for biopsy and histological conformation 6 . Because it can be mistaken with a malignant tumor, thus leading to unwarranted surgical removal, recognition of this lesion by different imaging techniques is of paramount importance ${ }^{7}$. Echocardiography is a noninvasive method used to diagnose intracardiac tumorous lesions, but it is not able to differentiate between fat and connective tissue ${ }^{8}$. Cardiac MRI is considered the imaging modality of choice for the assessment of cardiac masses. It provides high-quality, multiplanar imaging of tumor morphology, tissue composition, and perfusion. Additional advantages of cardiac MRI are the possibility of assessing cardiac function and blood flow, as well as its large field of view making it possible to visualize the paracardiac structures and the great vessels?.

This case shows an example of the potential appearance of cardiac involvement in ECD on echocardiographic images. Cardiac MRI is a valuable complement of echocardiography that provides tissue characterization and shows paracardiac structures that may be useful even in patients with a negative echocardiogram if cardiac involvement is suspected ${ }^{10}$.

In summary, in the present report, we described a case of ECD with cardiac involvement, presenting with symptomatic heart failure and a cardiac mass, assessed with echocardiogram and cardiac magnetic resonance imaging. 


\section{REFERENCES}

1. Masci PG, Zampa V, Barison A, Lombardi M. Cardiovascular involvement in ErdheimChester disease. Int $\mathrm{J}$ Cardiol. 2012;154(2):e24-6. http://dx.doi. org/10.1016/j.ijcard.2011.05.028. PMid:21676475

2. Bassou D, El Kharras A, Taoufik AT, En Nouali H, Elbaaj M, Benameur M, et al. Cardiac Erdheim-Chester. Intern Med. 2009;48(1):83-4. http://dx.doi. org/10.2169/internalmedicine.48.1448. PMid:19122364

3. Killu AM, Liang JJ, Jaffe AS. Erdheim-Chester disease with cardiac involvement successfully treated with anakinra. Int J Cardiol. 2013;167(5):e115-7. http://dx.doi. org/10.1016/j.jijcard.2013.04.057. PMid:23659884

4. Haroche J, Cluzel P, Toledano D, Montalescot G, Touitou D, Grenier $\mathrm{PA}$, et al. Images in cardiovascular medicine. Cardiac involvement in Erdheim-Chester disease: magnetic resonance and computed tomographic scan imaging in a monocentric series of 37 patients. Circulation. 2009;119(25):e597-

8. http://dx.doi.org/10.1161/

CIRCULATIONAHA.108.825075.

PMid:19564564

5. Raptis DA, Raptis CA, Jokerst C, Bhalla S. Erdheim-Chester disease with interatrial septum involvement. $J$ Thorac Imaging. 2012;27(5):W105-7. http://dx.doi. org/10.1097/RTI.0b013e31821db200. PMid:21681117

6. Xanthos T, Giannakopoulos N, Papadimitriou L. Lipomatous hypertrophy of the interatrial septum: a pathological and clinical approach. Int J Cardiol. 2007;121(1):48. http://dx.doi.org/10.1016/j. ijcard.2006.11.150. PMid:17292988

7. Calé R, Andrade MJ, Canada M, Hernandez-Estefania R, Lima S, Abecasis M, et al. Lipomatous hypertrophy of the interatrial septum: report of two cases where histological examination and surgical intervention were unavoidable. Eur J Echocardiogr. 2009;10(7):876-9. http://dx.doi.org/10.1093/ejechocard/ jep080. PMid:19525509

8. Heyer CM, Kagel T, Lemburg SP, Bauer TT, Nicolas V. Lipomatous hypertrophy of the interatrial septum: a prospective study of incidence, imaging findings, and clinical symptoms. Chest. 2003;124(6):206873. http://dx.doi.org/10.1378/ chest.124.6.2068. PMid:14665481

9. Fussen S, De Boeck BW, Zellweger MJ, Bremerich J, Goetschalckx $\mathrm{K}$, Zuber M, et al. Cardiovascular magnetic resonance imaging for diagnosis and clinical management of suspected cardiac masses and tumours. Eur Heart $J$. 2011;32(12):1551-60. http://dx.doi. org/10.1093/eurheartj/ehr104. PMid:21498848

10. Merli E, Savelli F, Lovato L, Zompatori M. Cardiac involvement in ErdheimChester disease: echocardiographic appearance and value of cardiac MRI. Eur Heart J Cardiovasc Imaging. 2012;13(2):198. http:// dx.doi.org/10.1093/ejechocard/jer236. PMid:22080452 